\title{
Paradoxa in the Blueprint of the Visual Organ: Their Contribution to 'Intelligent' Vision
}

\author{
Norbert Lauinger \\ Institut Für Optosensorik, Wetzlar, Germany \\ Email address: \\ norbert@lauinger-web.de \\ To cite this article: \\ Norbert Lauinger. Paradoxa in the Blueprint of the Visual Organ: Their Contribution to 'Intelligent' Vision. American Journal of Optics and \\ Photonics. Vol. 8, No. 2, 2020, pp. 40-50. doi: 10.11648/j.ajop.20200802.12
}

Received: June 30, 2020; Accepted: July 24, 2020; Published: August 13, 2020

\begin{abstract}
The development of the eyes is a product of the brain; the development of the central visual nerve tract which leads from the eyes to the brain is a product of the eyes and the development of the six extraocular muscles for the oculomotoric control of the eyes is a further product of the brain. Therefore, the human visual system is a product of the brain and the eyes. Paradoxical constructions are part of the blueprint. These are not self-explanatory and are not immediately recognizable as intelligent solutions. Some of these solutions have been labelled as a 'malperformance of nature', others are paradoxically misinterpreted. Some paradoxa are discussed before the grating-optical 'cortical' information processing in the three nuclear layers of the retina - the 'brain in the eye' - are looked at. The paradoxical construction of the visual nerve tract leading via the chiasm of the optic nerves (Chiasma Opticum) to the two CGL (Corpus Geniculatum Laterale) and to V1 (area 17 ) is based on this. At the same time, the 'brain in the eye' becomes the decisive basis of vision and the central visual nerve tract becomes a prominent organ of balance with a sensor and a motor function. Geometric optics and diffractive grating interference Near-field optics play an important part here as do the coordinate systems and the axis-centered symmetry operations.
\end{abstract}

Keywords: Human Vision, Inverted Human Retina, Nuclear Layers in the Retina, RGB-Vision in Daylight and Twilight, Monocular Depth Map, Brain in the Eye, The Eye an Organ of Balance

\section{Paradoxa and Intelligent Solutions in the Human Visual System}

The following can be considered to be paradoxical constructions: The cortical construction of two complementary hemispheres in the origin of the binocular visual system (1.1), the construction of two axes in each of the two eyes (1.2), the superiority of the development of the three nuclear layers in the retina of the eye before the development of the photo receptors (1.3), the determination of the luminous efficiency peaks of the photo receptors (cones and rods) in daylight and twilight vision (1.4), the development of a monocular depth map in the image space of each eye (1.5), the reduction of the information recipients from the cones (6.5 million) and rods (115 million) in daylight and twilight vision down to approx. 1 million visual nerves (1.6), vision with two eyes which do not add their luminosities (1.7). Based on these principles, the 'brain in the eye' (2.1) results with the fact that the luminosities of both eyes are not added playing a key role. Therefore, the gratingoptical diffractive near-field interference optics plays a decisive role in the explanation of vision. Furthermore, the axis-centered scheme of the visual nerves in the central visual nerve tract between both eyes and V1 (2.2) as an organ of balance in vision is discussed here.

\subsection{Complementary Hemispheres at the Cortical Origin of the Visual System}

The central z-body axis (Figure 1 left) develops at an early stage in the human embryo. It differentiates directly afterwards into the body's own $\mathrm{x}, \mathrm{y}, \mathrm{z}$-coordinate system (right).

Symmetrically identical to the central axis of the body's own coordinate system, two complementary hemispheres consisting of neuronal planar epithelium develop in the head part of the embryonic brain where several brain nerves originate at $2.6 \mathrm{~mm}$ embryo length. They grow out in 
opposite directions (Figure 2, left, $\mathrm{O}=$ ocular hemisphere). The hemispheres develop at an embryo length of $4 \mathrm{~mm}$ into eye stalks (Figure 2, right). The neuronal epithelium advances as far as the surface ectoderm (a). This earliest phase of the development of the binocular visual system is unusual due to the symmetry operation towards the central axis of the body's own coordinate system. Although this may initially appear to be paradoxical it leads already from the very beginning to a connection of the eyes to the brain as well as to an intelligent linking of both eyes to each other.
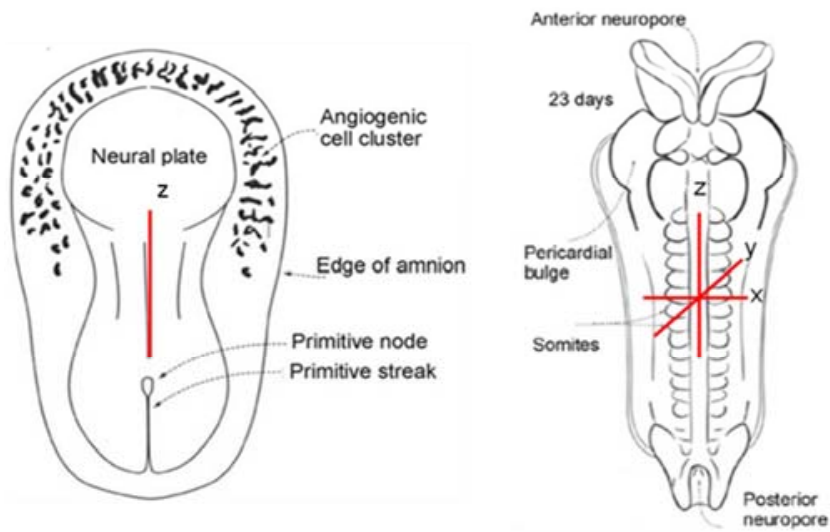

Figure 1. (left): the central body axis z develops in the initially planar embryo; (right) in the 23-day-old embryo the body's own three-dimensional $x, y, z$-coordinate system develops.
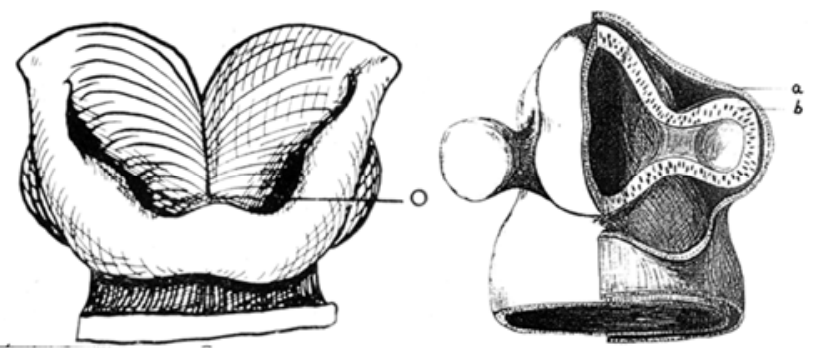

Figure 2. (Left) development of the eye in the forebrain halves $(2.6 \mathrm{~mm}$ embryo length) [I. Mann, 15, Figure 6, p. 7]. At $O$ and symmetrically in the second half of the brain, hemispheres grow out to both sides. (Right) The hemispheres develop in the 3rd-4th week into eye stalks [I. Mann, 15, Figure 17, p. 20]. In order to illustrate this better, the right half of the brain has been cut open. (C) 2013 reproduced with permission of the British Medical Association.

\subsection{One Optics with Two Axes and Two Poles: The Biaxial Bipolarity in the Retina}

Each eye has two axes (Figure 3) [Lauinger, 11]. Hardly any optician would probably consider the idea to choose such a paradoxical design for high-performance optics. However, what initially appears to be a paradox leads to an intelligent solution.

How can coaxiality and consequently bipolarity of the retina result? First of all, with the invagination of the frontal hemisphere of the retina's planar epithelium into the eye cavity (Figure 4), a part of the surface ectoderm which serves to construct the imaging optics (lens and retina) is introduced. In the inner eye, the invaginated neuronal planar epithelium fills the posterior space completely except for a narrow slit towards the pigment epithelium. This represents the basis of the retina.

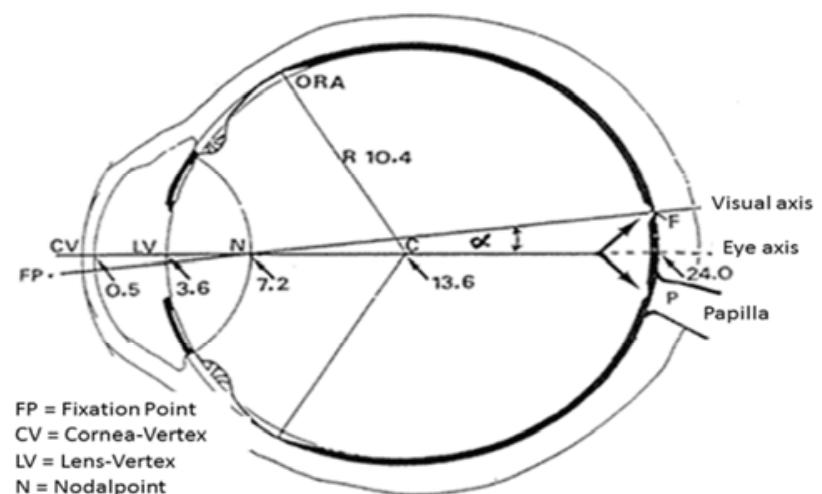

Figure 3. Primary eye axis and secondary visual axis in the human eye. Horizontal cut through the right eye with the nasal location of the papilla $P$ and temporal location of the fovea $F$. Angle $\alpha$ between the two axes. Distances from the cornea vertex are in $\mathrm{mm}$. The pivot point $C$ is located $13.6 \mathrm{~mm}$ behind the cornea vertex $\mathrm{CV}$.

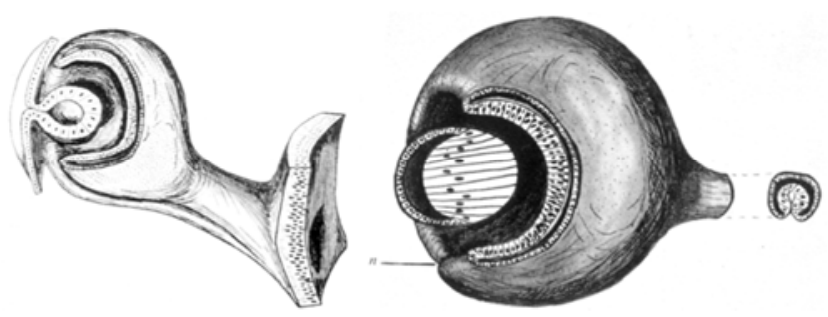

Figure 4. (Left) Invagination of a part of the surface ectoderm, (right) The lining of the eye cavity by means of the invaginated neuronal surface epithelium leads to the construction of the retina [I. Mann, 15].

While doing so, the inner eye is being centered to a first axis - the eye axis - in such a way that the optical nerves from all zones of the retina growing out of the ganglion cells of the inner nuclear layer of the retina can grow centripetally towards the zenith of the invaginated hemisphere. Initially however, no 'gate to the brain' through which the optical nerves might grow from the inner eye towards the brain is developed. However, such an opening is created quickly by an artery penetrating from outside into the inner eye (Figure 5 left) and in its zenith the subsequent papilla - the 'blind' spot - where no photo receptors can be stored later on is placed as the central pole in the central area of the retina. A blind spot in the zenith of the retina (Figure 5 right) can however under no circumstances be an acceptable solution for an eye.

A second step in the development starts with the storage of the macula in the central area of the retina - on the temporal side of the papilla and the eye axis. Figure 6 shows how the two eye developments are brought together by means of a reduction of the angles between the two eye stalks so that a widely overlapping central visual field is realized for the purpose of binocular vision. During pre-natal face development, the papilla is shifted in each eye towards the nose so that space is created for the storage of the macula on the side of the temples. 

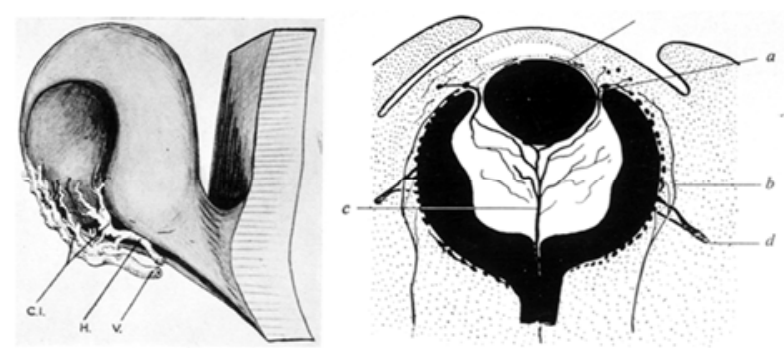

Figure 5. (Left) an artery penetrates the inner eye from below and takes up the central location in the zenith of the inner eye [I. Mann, 15].

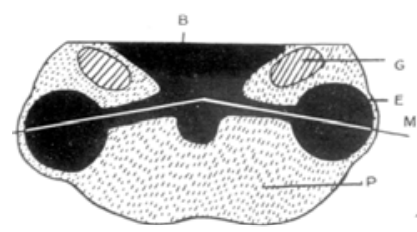

A. 9 mm. Angle $160^{\circ}$

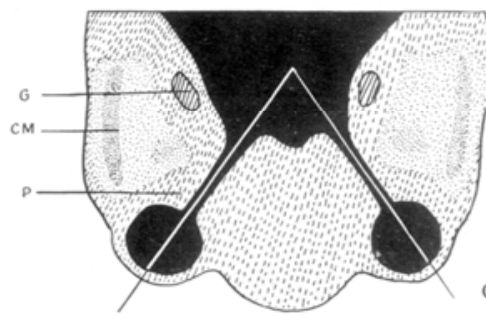

C. $40 \mathrm{~mm}$. Angle $72^{\circ}$
Figure 6. Pre-natal face development reduces the $160^{\circ}$ angle between the two eye constructions (at $9 \mathrm{~mm}$ length of the embryo) to $72^{\circ}$ (at $40 \mathrm{~mm}$ length of the embryo). With the development of the brow muscle ( $P$ in $B)$ during face development, the papilla in each eye is shifted towards the nose and temporally the macula is stored with a fovea as the center in each case [I. Mann, 15, p. 270].

The fovea as a retina hollow centers the cone and / or daylight vision and thus it introduces the second axis - the visual axis of daylight vision - which links the fixation point on the visual object with the fovea. Thus, a blind spot in the zenith of the inner eye is avoided and twilight vision with the elliptical zone of the highest density of rods remains centered to the two poles - fovea and papilla (Figure 7).

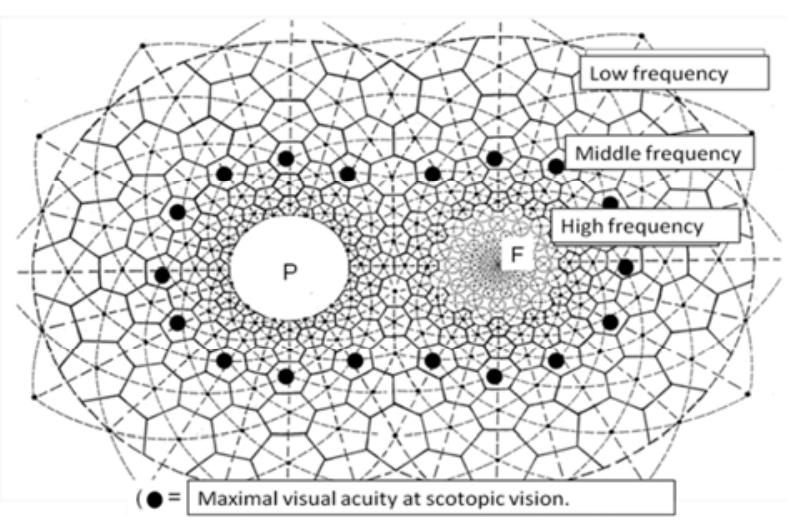

Figure 7. Papilla $(P)$ and Fovea $(F)$ make up the two poles of the retina, shown here for the right eye. The high-frequency image resolution (photopic visual acuity in daylight vision) displaces the scotopic curve of visual acuity on to the medium-frequency Cassini curve (scotopic visual acuity in twilight vision).
The intelligent result of the 2-axis construction can be seen in the fact that two types of vision with different photo receptors - cones and rods - are accommodated in the same organ. By means of a minor re-centering of the inner eye to the other axis, both types of vision resulting from subsequent pre-natal steps of development are functionally linked to each other. They both use the retinal network responsible for the values of visual acuity - the lateral structural resolution in the image. Only the peak values of visual acuity (1.0 in daylight vision and 0.4 in twilight vision) differ due to the macula storage in the central area of the retina. Thus, the paradox becomes an intelligent biaxial and bipolar solution in the image space of each eye.

\subsection{The Development of the Three Nuclear Layers in the Retina of the Eye Takes Priority over the Development of the Photo Receptors}

The invagination of the neuronal planar epithelium into the eye cavity represents the beginning of the development of the retina. Starting with the first nuclear layer which stores the ganglion cell nuclei, very rapidly two further nuclear layers differentiate in the space between the inner and outer limiting membrane of the retina [I. Mann, 15 and Blechschmidt, 4]. They are named the inner (INL), middle (MNL) and outer (ONL) nuclear layer. Paradoxically and at the same time falsely, they are considered as a so-called 'inverted retina' because they lie in a lightward direction on top of the photo receptor layer which does not follow until later. As a matter of fact, the three nuclear layers of cell bodies need to be considered as diffractive optical cell gratings (Figure 8) with the number of cells increasing in an outward direction and correspondingly decreasing grating constants, i.e. cell distances. The third nuclear layer which is represented in the fovea area needs to be assigned functionally to color vision in daylight vision. It represents a cellular diffractive space grating (with approx. 4-6 layers of cell nuclei). The association of the three nuclear layer-grating must be considered as a cortical part of the retina - as the 'brain in the eye'.

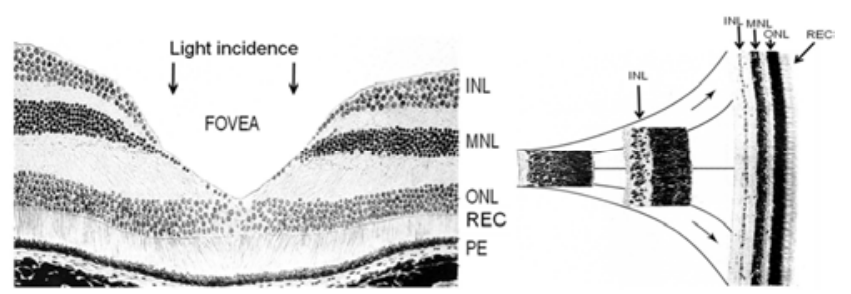

Figure 8. (left) Nuclear layers in the fovea area of the retina. INL = inner, $M N L=$ middle, $O N L=$ outer nuclear layer. $R E C=$ photo receptors (in the fovea only cones), $P E=$ pigment epithelium [Bargmann, 1]. (right) Differentiation of the three nuclear layers in the retina [Blechschmidt, 4, Figure 9]. (Left) undifferentiated gathering of cells, (middle) differentiation of the inner nuclear layer, (right) differentiation of the three INL, MNL and ONL layers with subsequent photo receptors growing only just before the end of the pre-natal development out of the ONL layer and positioned vertically to the nuclear layers. (C) 2013 with permission of S. Karger AG, Basel. 
The retina takes seven months to develop this cell grating hierarchy. Only then do the first photo receptors grow out of the cell nuclei of the ONL nuclear layer to penetrate through the outer membrane of the retina into the space behind the ONL layer in order to develop the distribution pattern of cones and rods there. With this cell grating hierarchy in the 'brain of the eye' thus far unconsidered functionalities in the eye itself arise leading not to the paradox of the 'inverted retina', but rather to an intelligent cortical performance.

\subsection{RGB-Color Vision with the Peaks of Three Spectral Luminous Efficiency Curves with $B=450 \mathrm{Nm}, G=535$ $\mathrm{Nm}$ and $\mathrm{R}=570 \mathrm{Nm}$ and Twilight Vision with One Single Peak at $512 \mathrm{Nm}$}

The basis of color vision is the transformation of the visible spectrum (380-760 nm) into RGB color space (REDGREEN-BLUE). The additive 3-color-composition in daylight vision is based on this with three types of cones as the basis according to common interpretation. Their photo pigments display spectral luminous efficiency peaks at 570 $\mathrm{nmG}, 535 \mathrm{nmG}$ and $450 \mathrm{nmB}$ [Rushton, 18] with a relatively large spectral half width (Figure 9). It may appear paradoxical to some opticians that with $450 \mathrm{nmB}, 535 \mathrm{nmG}$ and $570 \mathrm{nmR}$, the pigment chemistry in the outer segments of the cones should have chosen such a conspicuously asymmetrical basic setting of the photo-pigments in which the RGB-recipients would additionally first be linked locally by means of neuronal circuits. Moreover, the overlappings of the three curves have not been explained.
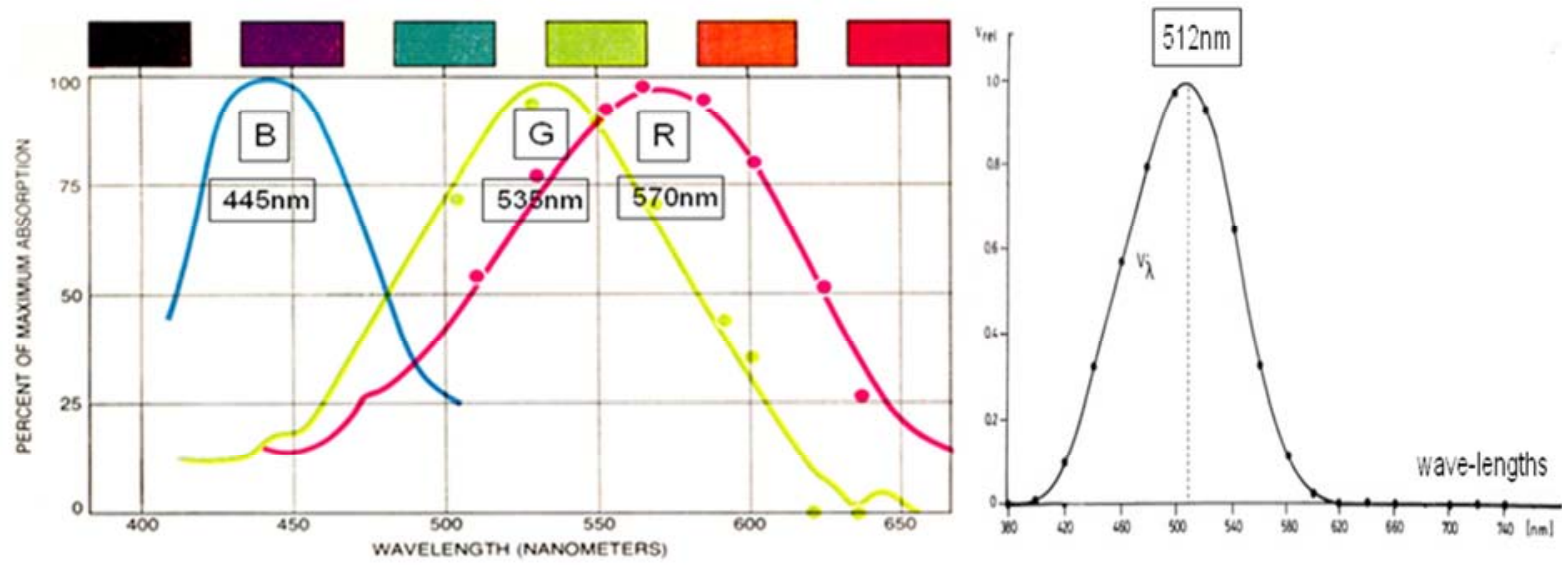

Figure 9. (left) The three luminous efficiency curves of the cones (daylight vision). (Right) Spectral luminous efficiency curve of the rods (twilight vision).

A more intelligent optical solution appears to be able to compete with this interpretation now [Lauinger, 13, 14]. It is based on the diffractive Fresnel near-field space grating optics in the outer cell body layer ONL (outer nuclear layer) of the retina and locally it forms three concentric optical diffractive orders with six interference maxima in each case at $559 \mathrm{nmR}, 537 \mathrm{nmG}$ und $447 \mathrm{nmB}$ (Figure 10) which are optically linked. With the RGB-sequence of the light cones there is also no overlapping of the diffraction orders. Furthermore, this optical solution also explains the spectral peaks of the luminous efficiency of the rod receptors via the merger of the $\mathrm{R}$ and $\mathrm{G}$ diffractive orders at $512 \mathrm{~nm}$ in the visible spectrum (Figure 9, right).

The so-called Purkinje shift, the transition from daylight (color) vision to twilight (luminosity) vision and vice versa is achieved by means of shortening and / or prolongation of the third grating constant of the space grating. Thus, the Purkinje shift becomes the result of a luminosity-adaptive optical process. The intelligent space grating optical solution which so far has only been considered mathematically, but not experimentally becomes recognizable behind the paradox. Optics - and not chemistry - and in particular the diffractive Fresnel near-field space grating optics used in crystal optics make the two points of view possible.

\subsection{Monocular Vision Without an Optical Depth Map}

The visible three-dimensional world in imaging optics is reduced to a planar two-dimensional world because to date there are only planar films or photo recipient arrays available. This represents a special trick of photographic optics. The reduction from $3 \mathrm{D}$ to $2 \mathrm{D}$ is achieved because the third dimension of the visible is processed in the aperture and / or lens space of imaging optics and not in its image space. Therefore, the photographer - being supported by an autofocus assistance - has to shift the imaging lenses in the optics when choosing the distance setting on the camera. In this way it is guaranteed that vision objects focused at different distances result in an acute image in the image space. If you close one eye in binocular vision, you can easily convince yourself that the three-dimensional visible world remains a three-dimensionally visible world and does not collapse into a 2-D view. Therefore, there is an optical depth map available in the image space of each eye. The lens-imaging equation in Figure 11 describes this fact. Distant vision objects are acutely imaged closer behind the eye lens and close objects further away behind the lens. If the normalsighted eye is set at a so-called accommodation-restingposition in relation to an object at a distance of $\mathrm{g}=5 \mathrm{~m}$, then the focal distance on the image side $\mathrm{f}^{*}=22.785 \mathrm{~mm}$ [Schober, 
20] and the acute image of this object will be at $b=22.889$ $\mathrm{mm}$. Then the acute image of a neighboring object located 10 $\mathrm{m}$ away with an unchanged $\mathrm{f}^{\prime}$ setting $\left(\mathrm{f}^{*}=22.785 \mathrm{~mm}\right)$ will be at $\mathrm{b}=22.837 \mathrm{~mm}$, thus $52 \mu \mathrm{m}$ in front of the image of the object $5 \mathrm{~m}$ away.

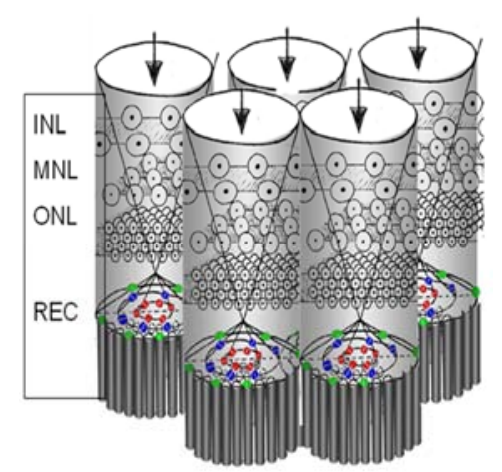

Figure 10. Hierarchy of the three nuclear layers interpreted as an optical grating with the grating constants reducing from the inside outwards and $R G B$-light cones in the photo receptor space behind the ONL-nuclear grating of the retina.

Objects closer to the eye are therefore principally imaged more acutely further away behind the eye lens than more distant objects. Therefore, someone living mainly in a nearworld with cellphones and computers from earliest youth onward will cause a prolongation of the eye in a backward direction and will due to this become short-sighted. The myopia-management will therefore have to ensure that all object distances are regularly processed in the 3-D space. If this fails, glasses and other visual aids are helpful.

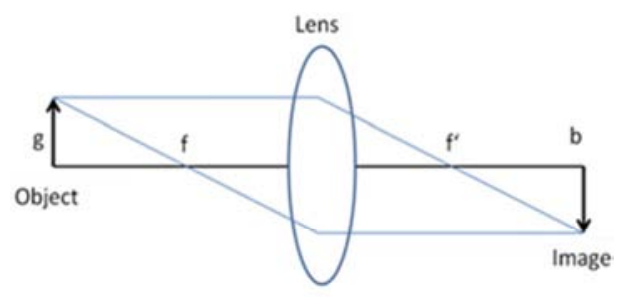

Lens Equation for the imaging with a thin lens

$$
\frac{1}{g}+\frac{1}{b}=\frac{1}{f} ; b=\frac{f^{\prime} g}{g-f} ; f=\frac{b g}{b+g}
$$

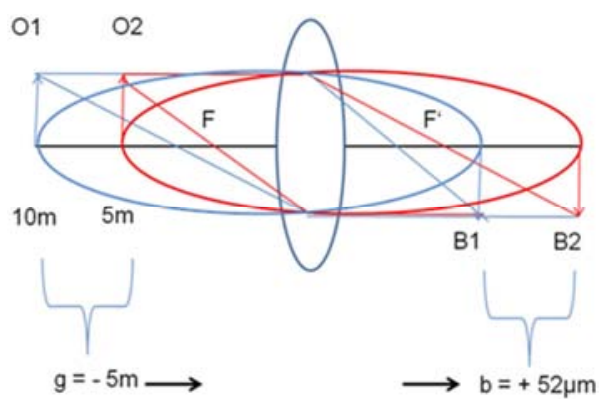

Figure 11. (upper picture) lens-imaging equation. (lower picture) object $(f)$ and image ( $f$ ') distances represent conjugated levels in the threedimensional space. With the focusing on objects $\mathrm{O} 1$ and / or O2 at different distances ( $g$ ) the image distance (b) changes. (Example: the reduction of the object distance from $10 \mathrm{~m}$ to $5 \mathrm{~m}$ results in a larger image distance of $52 \mu \mathrm{m}$ behind the lens.
Isn't it paradoxical to deny the existence of a 3-D depth map which is monocularly already available although in vision we certainly do not experience a shrinking of the world into a two-dimensional plane? Moreover, this happens despite its calculation by means of the lens-imaging equation. Photographic optics has led us astray. Intelligent 3-D optics has not been able to replace the paradoxical misbelief induced by photographic optics.

The geometric-optical depth map calculated by means of the lens-imaging equation can be further minimized in the image space by means storing two diffractive hexagonal gratings into the image plane (e.g. With a grating constant of $\mathrm{gx}=16 \mu \mathrm{m}$ in the first and of $\mathrm{gx}=8 \mu \mathrm{m}$ in the second grating). The difference of $52 \mu \mathrm{m}$ in the image space between the object which is $5 \mathrm{~m}$ and / or $10 \mathrm{~m}$ distant in the example above would then be shortened behind the 2 nd grating in a Talbot-1/4 plane to $28.6 \mu \mathrm{m}\left(\mathrm{z}=8 \mu \mathrm{m}^{2} / 4 \times 0.559 \mu \mathrm{m}\right)$ for a wavelength $\lambda=0.559 \mu \mathrm{m}$. (Three gratings with $\mathrm{gx}=16 \mu \mathrm{m}$, $8 \mu \mathrm{m}$ and $4 \mu \mathrm{m}$ for $\lambda=0.559 \mu \mathrm{mRed}$ would lead to a Talbot $/ 4$ depth map with $z=114.5+28.6+7.2=150.3 \mu \mathrm{m}$ which would correspond to an average retina thickness).

The example also shows that a reduction of the diffractive depth map will result in the retention of its third dimension and that it is not reduced to an image plane common to both objects. Decisive ways of playing with optical depth maps arise from such a geometric and diffractive-optical combination into which the three-dimensionality of the visible can be transferred. This is hugely different from the effort made by photographic optics.

\subsection{The Reduction of the Information Recipients to 1 Million Optic Nerves}

It is undisputed to this day and still as yet unexplained why there is a reduction of the information recipients of the cones (6.5 million) and rods (115 million) to approx. 1 million optic nerves in each eye in daylight and in twilight vision. The loss in image information which thus takes place through the central optic nerve tract to the cortical visual center V1 must be classified to be dramatic. Therefore, there must be a different reason for this reduction of data from the photo receptor pixel to the optic nerve basis. Also, information contents would be involved which would have been available for both daylight and twilight vision. It could be an acceptable solution that the pixel-details of the images would not be transmitted, but rather that the visual objects would be reduced to their conceptuality which in turn would be the optic answer to the question WHAT a visual object is generically: a house, a tree, a bird etc. The advantages would be: Terms are transmittable with less data, identical for daylight and twilight vision and for the cortex probably more important than enormous amounts of pixel-based data.

\subsection{Vision with Two Eyes Which Do Not Add Their Luminosities}

The fact that the luminosity is not halved when closing one eye represents another paradox in human vision. With the statement that the non-additivity of luminosities is an 
indication of interference processes, Optics [BergmannSchäfer, 2] provides the solution to the mystery. Diffractive Fresnel near-field optics in the cellular space grating of the retina follows the logic of wave optics. It makes vision more intelligent than photographic optics which follows the additivity of luminosities in the logic of geometrical optics. Fresnel near-field information processing is already used in telecommunications. In optics however, it is still limited in use.

\section{The 'Brain in the Eye' and the Optic Nerve Tract as an 'Organ of Balance'}

Following the analysis of some paradoxes in the blueprint of the eye and their contributions to vision the complete 'brain in the eye' and the 'visual system as an organ of balance' will now finally be evaluated.

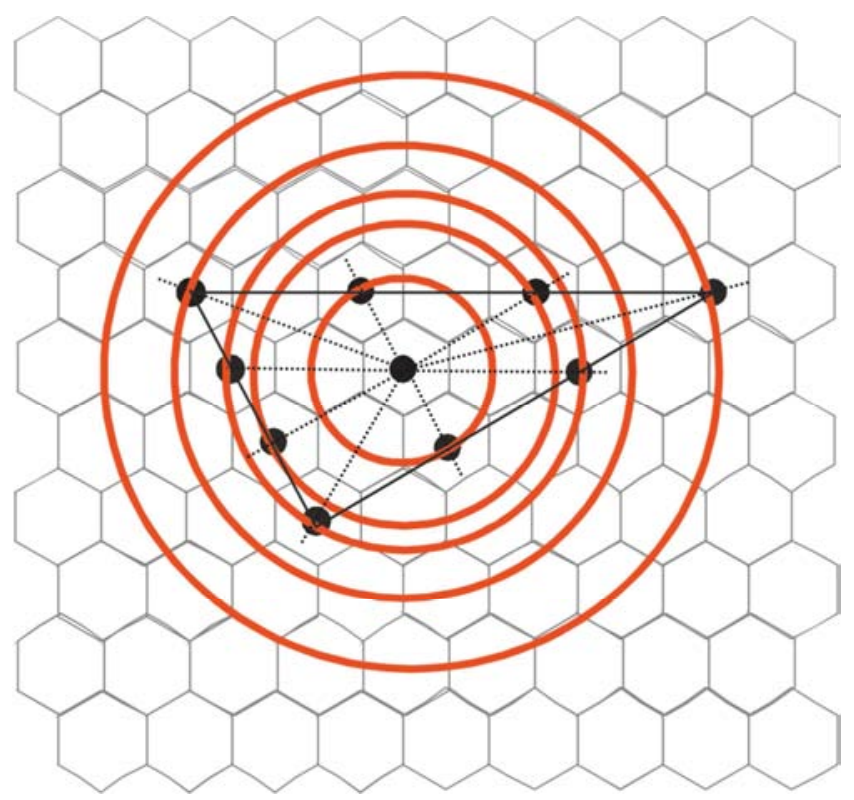

Figure 12. $R, \vartheta$-log-polar perception of the optical melody of a triangle in the hexagonal grating net. $r=$ radii of centered circles (5 circles), $\vartheta=$ angle of rotation $0-360^{\circ}$. Example for the perception of a focused triangle by means of nine polar vectors or $r, \vartheta$-values.

\subsection{The 'Brain in the Eye'}

The three nuclear layers between the limiting membranes of the retina represent the 'brain in the eye'. The inner and middle nuclear layer were already used functionally for the optical depth map in each eye; For the creation of RGB-color and / or brightness vision, the outer nuclear layer which provided a mathematically formulated contribution to daylight and twilight vision was used. Aside from the object distance and RGB-color and / or luminosity of the visual objects, 'intelligent' vision is only missing the development of the invariant 'optical melodies' of the visual objects which is analogue to the invariants in music. From vision the path leads to thinking [Glezer, 6] via the answer to the question "WHAT is something visible?" a house, a tree, a car etc... It leads from the optical image to the pre-lingual terms of the visual objects which are based mainly on the geometrical forms of visual objects. The terms are not named until later after language has been developed. Therefore, the 'cortical' analysis of the visible has to provide three important data: the relative distance of an object in the depth map and thus its separation from other objects or the background, its RGB-color or luminosity and its size-, orientation-and shift-invariant log-polar spatial frequency signature. A suitable instrument for the first step to abstract the conceptual term from the individual object form is the r, Э-log-polar perception of visual objects [Glezer, 6; Lauinger, 12] in the hexagonal net of the retina. Figure 12 illustrates this on an arbitrary triangle.

Figure 13 shows the $r$, $\vartheta$-diagram of the triangle. It demonstrates the conceptual abstraction - the 'melody' of the object.

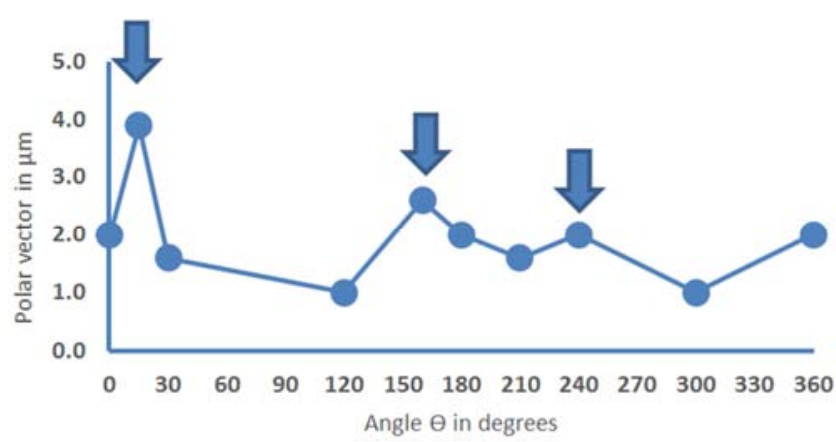

Figure 13. Diagram of the nine $r, \vartheta$-values of the triangle in Figure 12. The arrows mark the $r, \vartheta$-values of the three corners of the triangle.

Figure 14 represents the log-polar optical 'melody' of two different squares, with circle radii $r=\sqrt{27}$ up to $\sqrt{108} ; \vartheta=0^{\circ}$ up to $360^{\circ}$. Both squares are described by means of 12 common r-polar vectors and 69 -circles (only three of these have been drawn).

The log-polar r, 9-diagram of both squares in Figure 15 demonstrates the similarity of their 'melodies'. 22 polar vectors describe the large square and 20 polar vectors describe the small one. 12 polar vectors are common to both squares; 5 circles describe the small square and nine circles describe the large square. The arrows point to the position of the corners of the squares.

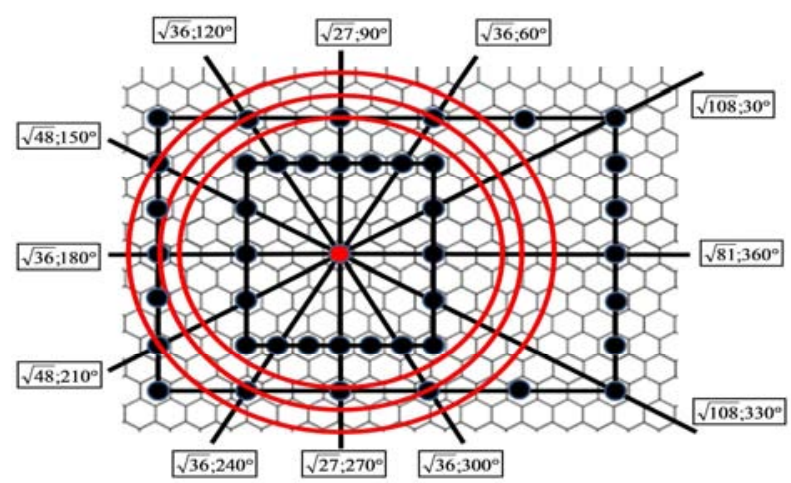

Figure 14. Two different squares with the same centering in the hexagonal net of the inner nuclear layer of the retina with grating constants $g_{x}=1 ; g_{y}=\sqrt{3}$ 


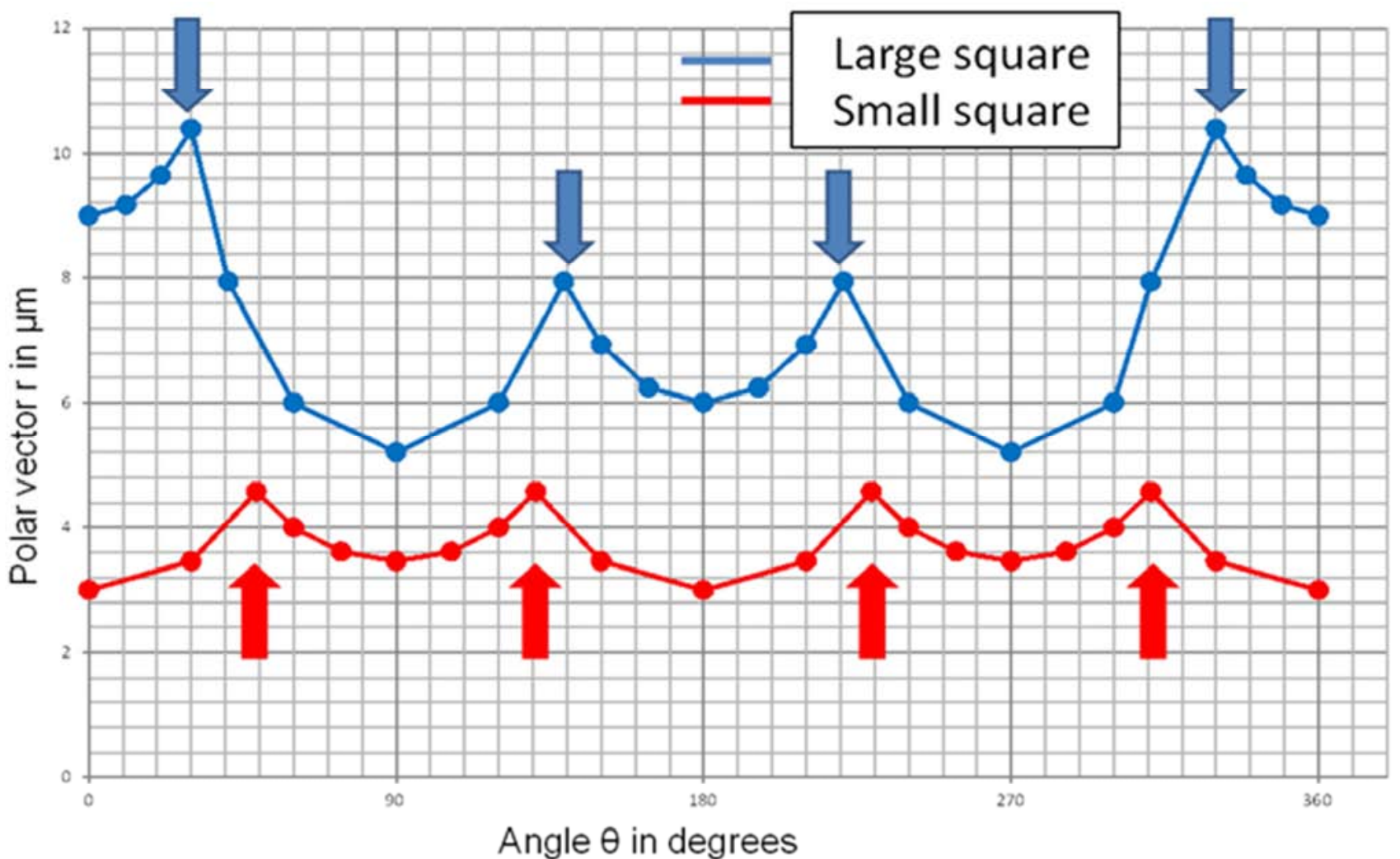

Figure 15. $r, \vartheta$-Log-polar perception of both squares in Figure 14.

Since the inner nuclear layer of the ganglion cells develops the basic net structure in vision with a high-frequency central mesh, a medium-frequency middle mesh and a low-frequency peripheral mesh, the inner nuclear layer INL with the local mesh structure of ganglion cell nuclei can take on the role of providing a centered resonance signature at the image location of a visual object by means of the log-polar spatial frequency analysis. In this way, the depth map, RGB-color or luminosity and the 'optical melody' of a focused object can be guaranteed by the 'brain in the eye'. All three nuclear layers as cortical cell gratings can contribute optically and simultaneously and still spatially in sequence. If the conceptual perception represents the highest priority in vision, then the depth map and RGB-vision are of subordinate importance. Regardless of distance and size, luminosity or RGB-color, a 'triangle' will always remain a triangle, even if it is perceived in daylight or twilight vision or in high-frequency or low-frequency retina areas. Figure 16 illustrates the corresponding division of roles of the retinal nuclear layers in image processing and the entry of the conceptual r, Э-object data into the optic nerves, either in the concentric signature or in the form of a diagram as Figures 13-15 illustrate.

The concept of photographic optics so far allows only the RGB-color analysis in the eye and shifts the third dimension as well as the conceptualization for a visual object into the cortex. It would then be exposed as a paradoxical notion. Does it not correspond to a paradox when the function of conceptualization in vision has to be relocated together with the depth map into the cortex because the 3-dimensionality of the visible and any conceptual object perception have been lost for the time being? The 'brain in the eye' offers a more intelligent solution. Currently it has only been explained in an initial approach what performances hierarchical optic gratings and space gratings in the Fresnel near-field image processing are able for and this would need to be researched thoroughly. Correlator optics [Haus, Lauinger, 7] demonstrated some innovative solutions by means of oscillating gratings in front of photo diode arrays. Following crystal optics in X-ray radiation, diffractive space grating optics is the next decisive and promising stage in optics development. An article by Ewald [Ewald, 5] has already announced this. Photonic crystals as optics based on space gratings and as data stores are a first step in this direction. The fact that image stabilization in saccades occurring in the case of a jumpy visual object or shifting views can be guaranteed by the retina and not the cortex [Idrees, 9] becomes even more probable by the possibility that instead of pixel data log-polar concepts of the visual objects can already be developed in the inner nuclear layer. 


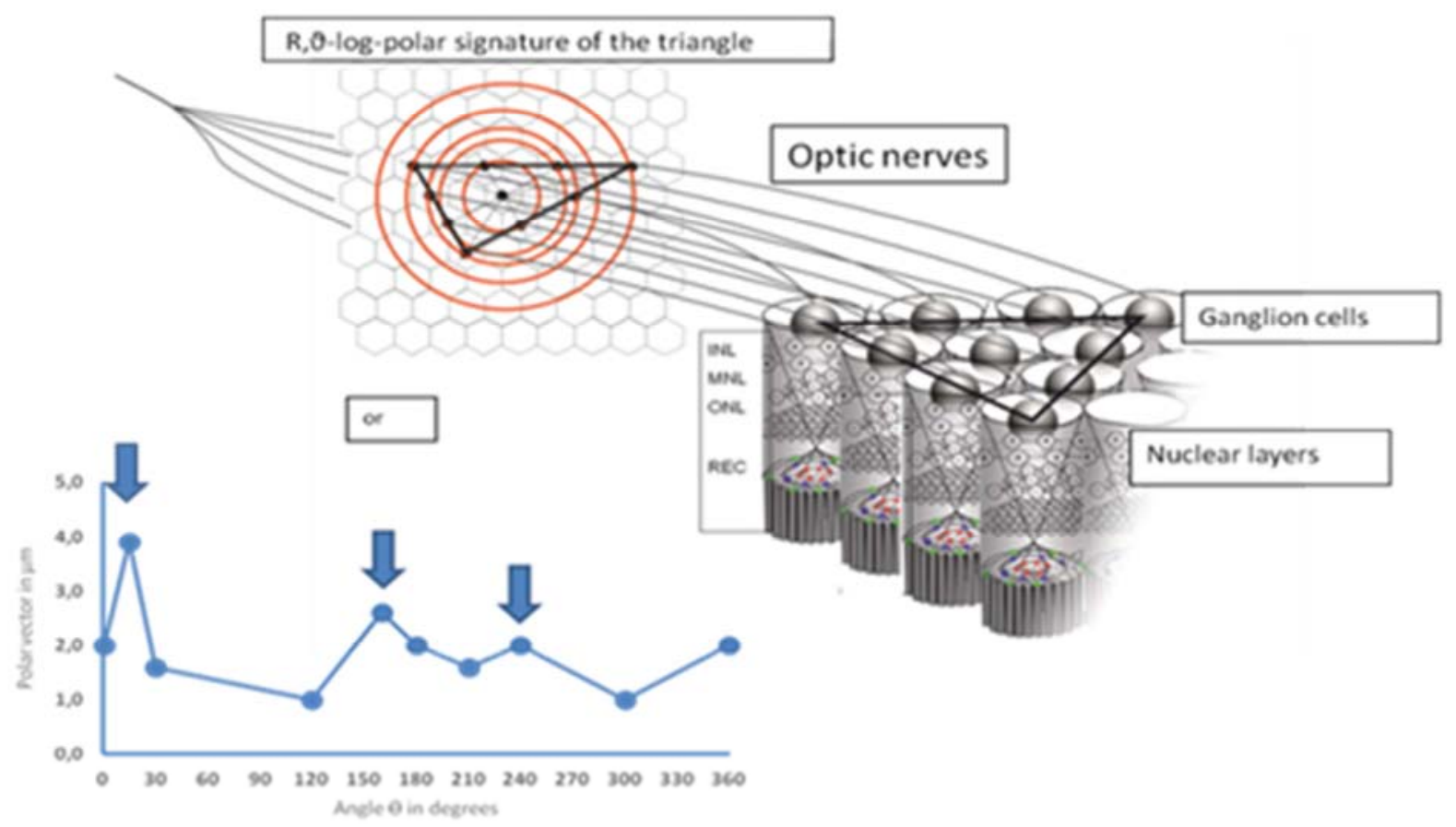

Figure 16. The division of roles of the retinal nuclear layers in image processing in the 'brain in the eye'. The INL layer of the ganglion cells in the retina provides the r, Э-log-polar conceptual 'melody' of the visual objects to the optic nerves. The INL and MNL layers provide the depth map of the visual objects; the ONL layer as a space grating produces the RGB-diffraction orders for daylight and twilight vision. These are at the disposal of the nano-antennas of the photo receptor outer segments for the optical-electrical transformation.

\subsection{The Visual System as an Organ of Balance}

If each of the two eyes contributes the 'cortical' performances of the 'brain in the eye' - the log-polar perception of the visual objects, the depth map and the RGBdata - then a merging and integration of these data in a central optic nerve tract from the eye to V1 can be advantageous in some respect. With one million optic nerves per eye the central optic nerve tract has the information situation in the inner nuclear layer of the retina at its disposal and it corresponds to that of the ganglion cells. When conceptual r, 9-log-polar signatures become available at the image location their data can be transferred directly into the optic nerves as data for the depth map or added to the RGBpixel data from layers located behind or from receptive fields of the ganglion cells. In this way, an explanation for the reduction to 1 million optic nerves would have been found. Traces in the CGL and in V1 prove that this does not necessarily mean that the optic nerves have to forego data from the deeper retinal layers. It is clear from the beginning that the central optic nerve tract has other goals than just a data transfer from both image spaces of the eyes into a common center V1. It links the coordinate systems of the eyes to one another and to the body's own coordinate system of the brain and with this double linkage it changes the organ of vision into an important organ of balance.

Development of the optic tract begins in the retina of each eye at a very early stage of pre-natal development. It leads from the retina via the optic chiasm (Chiasma Opticum) to the centers on either side CGL (Corpus Geniculatum Laterale) and from there to the cortical center of vision V1.
In the left Corpus Geniculatum Laterale (CGL) the left half of the retina of the left eye is merged with the left half of the retina of the right eye and both halves are superimposed on each other in up to 6 layers. The same happens in the right CGL with the right halves of both retinas.

Ramon y Cajal described [Cajal, 17] how this development in binocular vision with overlapping fields of vision has to obligatorily lead to a chiasm of the optic tract in order to guarantee the identity of a visual object in V1. Figure 17 (left) illustrates this issue. Figure 17 (right) shows schematically the division into hemispheres and quadrants in the optic nerve tract of an eye as has been proven during the lesion of certain sections in the case of pathological vision failure [Purves, 16, Figure 11. 8, p. 268]. In both parts of the Figure 17 it cannot be ignored that the design of the course of the optic nerves corresponds to an axis-centered symmetry operation. The central axis corresponds to the z-axis in the body's own coordinate system linking V1 with the fixation location on the visual object.

The optic nerve tract serves the linking of both eyes to each other and of the eyes to the brain in monocular as well as binocular vision. It is a product of the ganglion cells whose axons grow out of all retina zones in the inner nuclear layer of the retina radially to the papilla - the gateway to the brain and later on the 'blind spot' in vision. The initial radial course of the optic nerves is modified by means of the late and temporal storage of the macula into the central area of the retina. The papillo-macular bundle of optic nerves in Figure 18 illustrates the late access to the papilla and thus to the complete optic nerve of each eye for the right eye. The 
first and decisive result in the organization of the bundle of optic nerves is its division into quadrants. Hogan-AlvaradoWeddel [8, p. 535] provided electron-microscopical proof for this. For the two axes upon which the division into quadrants in the retina is based it can be asserted: „The vertical and horizontal lines divide the retinal ganglion cells into four quadrants". It also holds. "The ganglion cells in each quadrant are responsible for vision in that quadrant, including the macula and fovea". Thus, each eye with approx. 1 million optic nerves develops its part in the central optic nerve tract to the brain at a very early stage. The visual axis in the eye linking the fovea with the fixation point on the visual object represents the third axis in the Cartesian coordinate system of the eye. Correspondingly, this also holds true for the visual axis in twilight vision. The course of the optic nerve tract in both eyes are represented by symmetrically identical Figures. The papilla is always located towards the nose and the fovea towards the side in the eye.

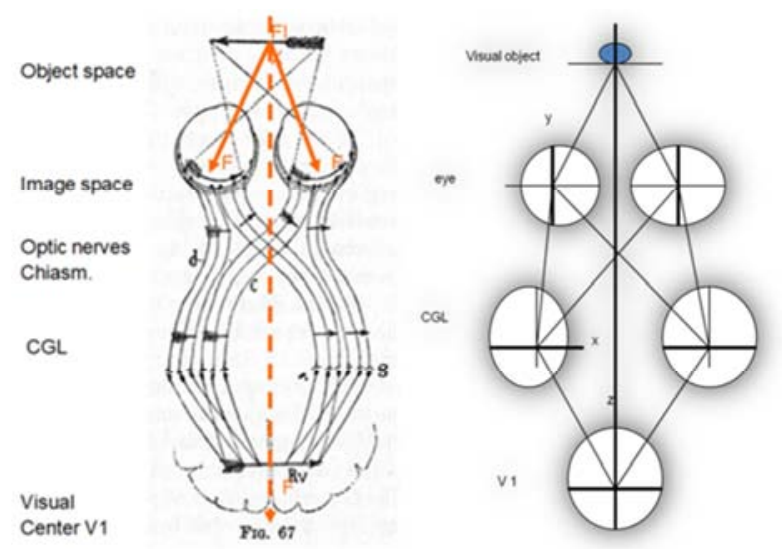

Figure 17. (left) The binocular development compels the optic chiasm in order to guarantee the identity of the visual object in V1 (Ramon y Cajal [17, Figure. 67, p. 473]. FIX = Fixation point visual object, $F=$ Fovea. The visual axes linking the fixation point on the object with the fovea of the eyes was added by the author as well as the central axis drawn as a broken line. (Right) Schematic illustration of the symmetry operations in the central optic tract based on the division into squares (x. y. z axes). The cortical z-axis links the fovea in V1 to the fixation point on the binocularly targeted object.

The question needs to be posed how the development of a coordinate system in each eye comes about. The horizontal and vertical line of the retinal division into quadrants results from the projection of the Cartesian coordinate system which is developed in each eye and consists of three axes $\mathrm{x}, \mathrm{y} . \mathrm{z}$ into the image space. "Since the rotational point remains nearly unaltered during all eye movements" [Berke, 3, p. 20] "all eye movements can be reduced to rotations around three axes. Therefore, such a "3-D coordinate system is necessary" for the description of eye movements based on the rotational point of the eye [Berke, 3, p. 20]. "The 3 coordinate axes are also called Fick's axes. The $\mathrm{x}$-axis and the z-axis are located frontally-parallel in Listing's plane with the z-axis being vertical and the $\mathrm{x}$-axis running horizontally. The $\mathrm{y}$-axis forms the vertical axis on to this plane in the rotational point of the eye. All movements of the eye are related to these coordinate axes" [Berke, 3, p. 20]. Therefore, from the very beginning each eye has a 3-D coordinate system usable in a sensory and motoric fashion. Basically, the importance of a 3-D coordinate system lies in the fact that places in the visual space in the 3-D reference system of each eye can be clearly localized.

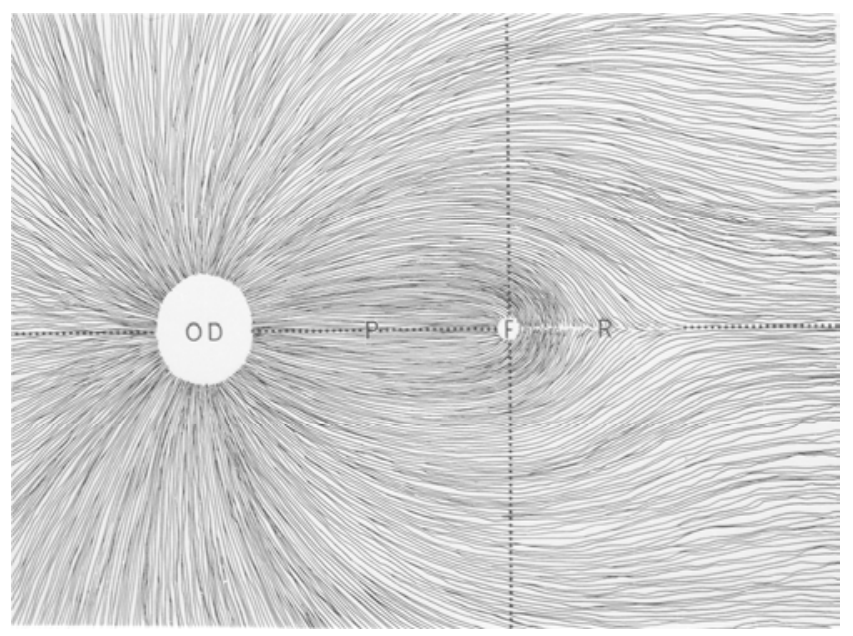

Figure 18. Figure showing the course of the optic nerves in the central area of the retina of the right eye. The papillo-macular bundle of optic nerves is stored in the original radial Figure of the course of the optic nerves. In the fovea the division into quadrants is being developed. $(O D=$ Papilla (optic disc), $F=$ Fovea, $P=$ papillo-macular bundle, $R=$ Raphe-dividing line) [Hogan, Figure 10-8, p. 536].

Figure 19 illustrates that the link of the coordinate system of the eye to the body's own coordinate system is of decisive importance. The left part of the Figure shows the case in which the object targeted by both eyes is located on the central axis of the body's own coordinate system. In the right part of the Figure the orientation of the body's own z-axis remains, but the eyes shift their coordinate system on to another object. Without a direct reference of the eyes' 3-D coordinate system to the body's own coordinate system there can be no effective control of movement in space. With the central optic tract providing this reference, it meets the requirements of the role as an organ of balance in the optic system. The path of the optic nerve bundles being cut into quadrants only appears to be paradoxical, but with the mirrored contents of the images it reveals an intelligent solution here also.

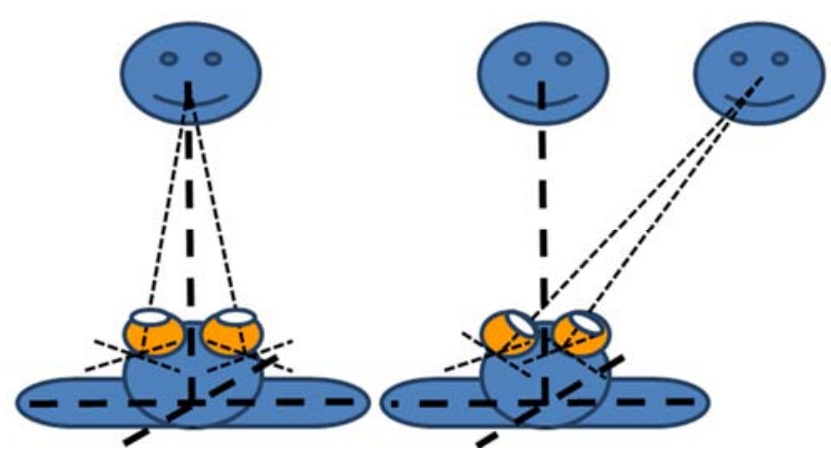

Figure 19. Identical (left) and unidentical (right) coordinate systems: Coordinate system in the eye; Coordinate system in the head. 
Since also the inner ear captures the three dimensions of the space in a 3-D coordinate system in a sensory manner by means of three vertically stacked arcades of the vestibular apparatus and reports changes in speed like movements of the eyes to the cerebellum, finally all senses support the coordination of the 3-D coordinate systems. The basis of the organ of balance in vision lies in the interaction of the coordinate systems necessary for vision in one and / or both eyes with the body's own xyz-coordinate system anchored in $\mathrm{V} 1$ and / or in the cortex. If one eye fails for whatever reason, the other will develop a correspondingly reduced central optic tract on its own to become a monocular organ of balance. For practical purposes, it has been seen that astronauts disembarking after landing or persons returning from the virtual reality world into the real world [Schindler, 19] have to rely enormously on the visual system in each of the functionalities shown. Only this interpretation of the optic tract makes that understandable which astonishes every optician because for them it remains incomprehensible what sense there is in cutting up and separate pathways of images provided by the eyes of centrally perceived objects in four quadrants.

\subsection{The Final Grasp of the Brain for the Eyes in Order to Control the Oculo-Motoric Function}

Temporally parallel to pre-natal development of the optic tract, in each case six extra-ocular eye muscles develop in the background of the ocular globes with neuronal tracts to the brain stem as illustrated in Figure 20 for one eye. By means of this connection of the eyes to the brain the steering and control of eye movements - the so-called oculo-motoric control is achieved. The anchoring of the muscles in the sclera of the eyes enables an extremely ingenious steering of the eye movements. The central optic nerve tract of each eye is not obstructed by this on its way to the optic chiasm. By means of focusing of a visual object an optical accommodation as regards the object distance occurs. The symmetry operation of both eyes again corresponds to a mirroring centered on the axes with the reflection axis being the direct connecting line from V1 to the fixation point on the visual object.

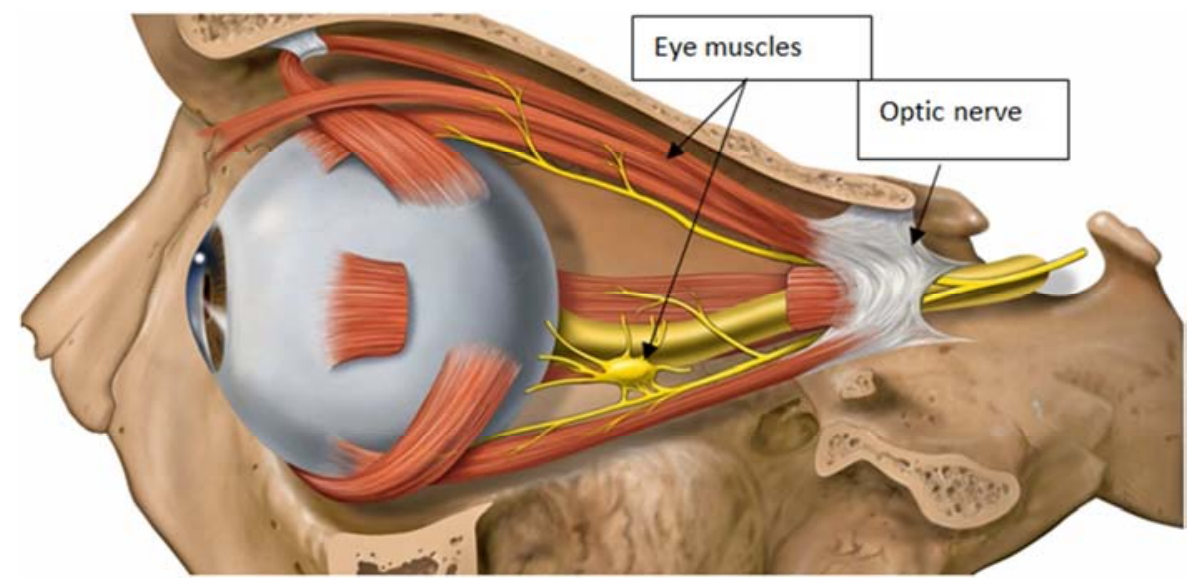

Figure 20. The brain stem grasps the eye balls from behind during pre-natal development. https://commons.wikimedia.org/wiki/File:Lateral_orbit_nerves.jpg

Since the renewed linking of the eyes to the brain and the oculo-motoric control resulting from this have been described in depth, the reference regarding the corresponding literature may suffice here [Kaufmann, 10, Kap. 35+36].

\section{Summary}

Image processing and visual orientation in space represent two different, but important functionalities for the human organ of vision. The cooperation between the brain and the eyes in linked coordinate systems is based in the pre-natal development of the visual system with intelligent solutions hiding behind paradoxical steps of development. They practice this until far beyond the post-natal period. It becomes clear immediately that the requirements and achievements of vision go far beyond the concept of photographic optics which offers no solutions for a depth map in the image space of an imaging optics and neither does it have a conceptual perception of visual objects and therefore it has to process this in software nets subordinate to optics. The interference-optical Fresnel near-field processing of the visible in micro-gratings and space gratings remains an open field for the development of diffractive optics such as future concepts for retina implants. The 'brain in the eye' provides the basis for 'intelligent' vision. In this, a clear hierarchy of priorities in visual object recognition and / or image processing results. First of all, there is the prelinguistic answer to the question as to WHAT something visible is, the invariant 'melody' or conceptualization of the visible; Secondly come the spatial distances between visual objects, the depth map information followed in thirdly by the RGB-color and / or luminosities of the objects. The combination of imaging optics with the space grating optics provide all three characteristics in the 'brain in the eye' and its subordinate nano-antennas photo receptors. For robotics development, a new setting of priorities results in the image space of an optical imaging system for the purpose of simultaneous availability of conceptual perception of the visual objects prior to their depth map and prior to their RGB-color and / or luminosity. 


\section{References}

[1] W. Bargmann, Histologie und mikroskopische Anatomie des Menschen. Verlag Thieme, Stuttgart, 1967.

[2] L. Bergmann, C. Schaefer, Lehrbuch der Experimentalphysik, Band III, Teil 1 Wellenoptik, Walter de Gruyter \& Co, Berlin $(2 / 1959)$ und Band III Optik, Walter de Gruyter \& Co (8/1987).

[3] A. Berke, Augenmuskeln und Augenbewegungen. Optometrie 1/2000, 13-27. PDF im Internet unter „Augenmuskeln“.

[4] E. Blechschmidt, Die Entwicklungsbewegungen der menschlichen Retina zur Zeit der Irisentstehung. Ophthalmologica, Vol. 154, 1967, pp. 531-550.

[5] P. P. Ewald, Crystal optics for visible light and $\mathrm{x}$ rays. Reviews of Modern Physics, 37 (1), 1965, pp. 46-56.

[6] V. D. Glezer, Vision and Mind. Modeling Mental Functions. Lawrence Erlbaum Associates, Mahwah New Jersey, 1995.

[7] J. Haus, N. Lauinger, Optische Gitter: Die Abbildung der Realität-75 Jahre berührungslose dynamische Messtechnik auf der Basis optischer Gitter. Laser Technik Journal, 4: 2007, pp. 43-47.

[8] M. J. Hogan, J. A. Alvarado, J. E. Weddell, Histology of the Human Eye. W. B. Saunders, Philadelphia-London-Toronto, 1971.

[9] S. Idrees et al., Wie der ,Bildstabilisator ' in unseren Augen funktioniert. https://www.wissenschaft.de/gesundheit-medizin, Universität Tübingen, 28. 04. 2020; dgl. In Nature Communications, doi: 10.1038/s41467-020-15890-w.

[10] P. L. Kaufmann, A. Alm; Adler's Physiology of the Eye., Mosby, 10/2003, Section 14, 34-36, pp. 787-858, Extraocular Muscles/Eye Movements.

[11] N. Lauinger, The two axes of the Human Eye and Inversion of the retinal layers: the basis for the interpretation of the retina as a phase grating optical cellular 3D chip. Journal of Biological Physics 19, 1994, 243-257.

[12] N. Lauinger, The Human Eye: An Intelligent Optical Sensor (The Inverted Human Retina: A Diffractive-Optical Correlator). IFSA International Frequency Sensor Association Publishing, Barcelona, 2014.

[13] N. Lauinger, Space grating optical structure of the retina and RGB-color vision. Applied Optics, Vol. 56, No. 4, 2017, 12611265 .

[14] N. Lauinger, Fresnel Nearfield Space-Grating Optics in the Human Retina Explains Human Color and Dimlight Vision. S. Y. Yurish Editor, Advances in Optics: Reviews. Book Series, Vo. 1, IFSA Publishing, 2018, pp. 415-435.

[15] I. Mann, The Development of the Human Eye, $2^{\text {nd }}$ Edition British Medical Ass. London 1949.

[16] D. Purves, G. J. Augustine, D. Fitzpatrick, WC. Hall et al. Neuroscience. Sinauer Assoc., 3/2004.

[17] S. Ramon y Cajal, Recollections of My Life, MIT Press, 1996.

[18] W. A. H. Rushton, Visual Pigments and Color Blindness. Scientific American, 232, 1975, 64-74.

[19] A. Schindler, A. Bartels, Verrechnung visueller Wahrnehmung bei Kopfbewegungen, Zeitschrift Augenspiegel für Klinik und Praxis, Forum für Augenärzte, April 2018, p. 6.

[20] H. Schober, Das Sehen. VEB-Verlag Leipzig, 4/1970. 